\title{
FOUR NEW EVOLVED PLANETARY NEBULAE
}

\author{
S. TAMURA \\ Astronomical Institute, Tohoku University, Aoba-ku, Sendai 980, Japan \\ and \\ R. WEINBERGER \\ Institut für Astronomie, Universität Innsbruck, Technikerstr. 25, Austria
}

While examining Palomar Observatory Sky Survey prints for various purposes, we came upon a number of hitherto uncatalogued nebulous objects, all of them of low surface brightness. Four of them are considered by us as new planetary nebula candidates due to their morphology. For the brightest one of them, spectroscopic observations were carried out with the Cassegrain spectrograph attached to the 74inch telescope of the Okayama Astrophysical Observatory: this object $\left(1=65.49^{\circ}\right.$, $\left.\mathrm{b}=+3.18^{\circ}\right)$ is clearly confirmed as a planetary nebula and obviously is in an advanced stage in its evolution; in Fig. 1, a spectrum of it is shown.

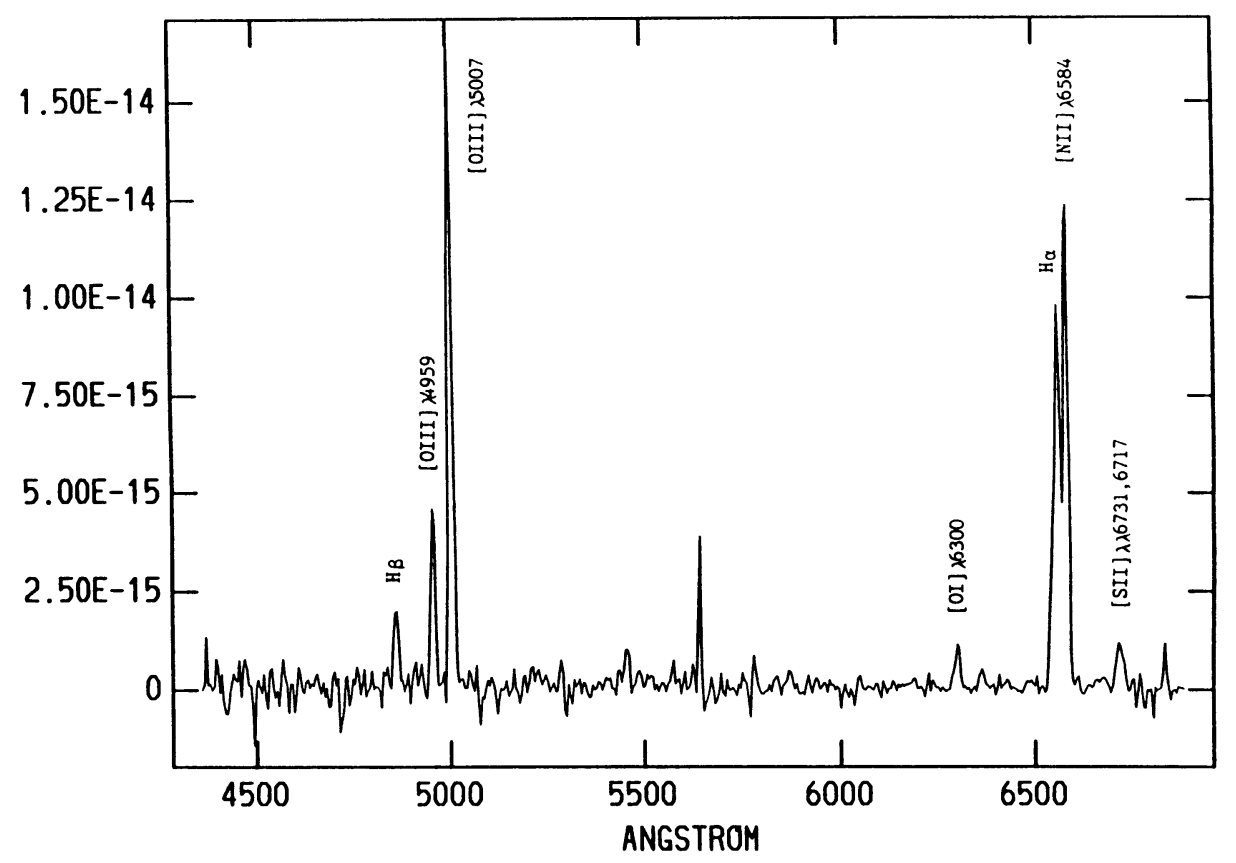

Fig. 1. The low-dispersion spectrum of the new PN at $\alpha=19^{h} 41^{m} 49^{s}, \delta=+30^{\circ} 06^{\prime} 49^{\prime \prime}$ 